\title{
Association of $A B O$ Blood Grouping and Periodontal Disease: A Literature Review
}

\author{
Ghani Ur Rehman ${ }^{10}$ \\ 1 Yunnan Key Laboratory of Primate Biomedical Research, Institute of \\ Primate Translational Medicine, Kunming University of Science and \\ Technology, Kunming, Yunnan, People's Republic of China \\ Dent J Adv Stud 2021;9:116-120.
}

\begin{abstract}
Address for correspondence Ghani Ur Rehman, PhD, Yunnan Key Laboratory of Primate Biomedical Research, Institute of Primate Translational Medicine, Kunming University of Science and Technology, 650500, Kunming, Yunnan, People's Republic China (e-mail: ghanigenetics@gmail.com).
\end{abstract}

\begin{abstract}
Keywords

- periodontal disease

- PD

- gingivitis

- ABO blood groups

- aggressive periodontitis

Although the correlation between the $\mathrm{ABO}$ blood group and certain systematic diseases is a well-documented fact. Few studies have explored the relationship of $A B O$ with oral disorders including periodontal diseases (PD). This literature review aimed to assess the possible association between $A B O$ blood grouping and PD. For this purpose, we searched online databases like PubMed and google scholar with the following terms in different combinations $\mathrm{PD}, \mathrm{ABO}$ blood groups, gingivitis, the prevalence of periodontitis, aggressive periodontitis, risk factors of periodontitis, Association of $\mathrm{PD}$ and $\mathrm{ABO}, \mathrm{ABO}$ Rh factor. English version papers describing the Oral health status are covered through research that includes clinical investigations, case studies, and retrospective studies on the subject. Historic reviews, articles with unpublished data, and letters to the editors were omitted from the survey following publications. The author thoroughly checked the titles and abstracts of the papers. The complete text was thoroughly assessed, and the relevant articles were analyzed in accordance with qualifying criteria. For further original and review research, references were further reviewed. Since our targeted subject was answered by a restricted number of original research. We identified fourteen articles describing the association of $A B O$ blood types with PD. findings of our study concluded the genetic basis of the association of $\mathrm{ABO}$ blood types for the development of PD. six of the research provided here showed that the blood type $O$ can serve as a prognostic factor for the development of PD. ABO blood types and Rhesus factors can be a risk predictors in the development of PD.
\end{abstract}

\section{Introduction}

Periodontal disease (PD) is a broad term that refers to many chronic inflammatory conditions affecting the gingiva bone and ligament that supports the teeth. It includes alveolar bone inflammation, gingiva, and periodontal ligaments. ${ }^{1}$
Primarily, bacterial infection is the main cause of PD, which results in localized gingival inflammation produced by bacteria in dental plaque, a microbial biofilm that accumulates on the teeth and gingiva. When left untreated, PD can result in the deterioration of the structure around the teeth. $^{2}$ published online November 22, 2021
DOI https://doi.org/ 10.1055/s-0041-1736487. ISSN 2321-1482.

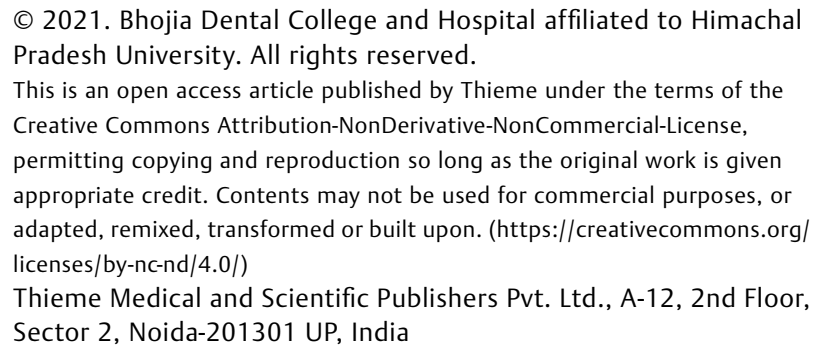

(c) 2021. Bhojia Dental College and Hospital affiliated to Himachal Pradesh University. All rights reserved.

This is an open access article published by Thieme under the terms of the Creative Commons Attribution-NonDerivative-NonCommercial-License, permitting copying and reproduction so long as the original work is given appropriate credit. Contents may not be used for commercial purposes, or adapted, remixed, transformed or built upon. (https://creativecommons.org/ licenses/by-nc-nd/4.0/)

Thieme Medical and Scientific Publishers Pvt. Ltd., A-12, 2nd Floor, Sector 2, Noida-201301 UP, India 
The untreated gingivitis proceeds to the loss of gingiva, bone, and ligament, chronic periodontitis (CP) develops, resulting in the deep periodontal pockets that are a characteristic of the illness and can eventually lead to tooth loss. PD may have a role in the body's total inflammatory load, and this might potentially worsen other conditions such as diabetes mellitus and atherosclerosis. ${ }^{3-5}$

Findings concerning epidemiological studies that apply continuous assessments of probing depth and clinical attachment loss have discovered that $10-15 \%$ of the worldwide population is plagued by advanced forms of periodontitis. ${ }^{6}$ Both severe aggressive periodontitis (AP) and severe $\mathrm{CP}$ are included in this predicted prevalence range. ${ }^{7,8}$ The number of bacteria in plaque does not appear to be highly correlated with the severity of PD, and some studies have postulated that hereditary influences may play a role. ${ }^{7-9}$

It was Karl Landsteiner who originally credited the ABO method in 1900. Blood type is based on the presence or absence of $A$ and $B$ surface antigens. According to the blood group classification, blood type is divided into groups $A, B, O$, and $A B$, each representing the presence or absence of different types of antigens red blood cells (RBCs). ${ }^{10}$ Additionally, our understanding of blood groups has expanded to include not just transfusion-related difficulties but also the possibility of an individual developing a specific disease due to RBC surface antigens. Another notable example is that individuals who belong to the group A blood type have been associated with an increased risk of gallstones, ulcerative colitis, and some types of tumor, ${ }^{11}$ whereas the other blood group has been associated with certain cardiac disorders, including ischemic heart disease and atherosclerosis. ${ }^{12-15}$ Only a few researchers have studied the possible relationship of $A B O$ with oral disorders notably PD.,16,17 The purpose of the present literature review was to find out if there is any association between the ABO blood type and PD, and what processes may be responsible for the association.

\section{Materials and Methods}

The primary goal of this review was to see if there was any possible association between PD and ABO blood type. Several online databases, including Google scholar, PubMed/MEDLINE, and SciHub, were used to address this query. The desired articles found in the database were searched from 1975 to 2017 using a variety of combinations of the words listed as PD, ABO blood groups, gingivitis, the prevalence of periodontitis, AP, risk factors of periodontitis, association of $\mathrm{PD}$, and $\mathrm{ABO}, \mathrm{ABO} \mathrm{Rh}$ factor. English version papers describing the oral health status were covered through research that included clinical investigations, case studies, and retrospective studies on the subject. Historic reviews, articles with unpublished data, and letters to the editors were omitted from the survey following publications.

The author thoroughly checked the titles and abstracts of the papers. For further original and review research, complete texts were thoroughly assessed, and the relevant articles were analyzed as per qualifying criteria. Reference lists were software reviewed. Since the targeted subject was answered by a restricted number of original research, this review was designed to synthesize the appropriate information.

\section{Results}

Fourteen research have been retrieved from the online literature. The key elements of each study are summarized in -Table 1.

\section{Relationship of ABO with the Incidence of Aggressive Periodontitis}

However, despite AP is frequently found in healthy individuals, ${ }^{18}$ a few studies have indicated that it has been linked to numerous hereditary disorders. A compromised immune response weakened by disease on the host is a critical risk factor in the development of severe types of periodontitis. ${ }^{19}$ Some systemic diseases can affect the host immune system and are typically linked to destructive PD. ${ }^{19}$ It has been speculated that human leukocyte antigen HLA A9 and/or B15 is a potential marker for AP. The risk of AP was 1.5 to 3.5 times higher comparing those who do not carry HLA antibodies. $^{20}$

In this review, five of the papers studied the association of the AP and ABO blood types, which analyzed an improved incidence of AP in B blood group, ${ }^{21}$ while in blood group 0 there was a decreased incidence of AP. A study, association of $\mathrm{ABO}$ with juvenile periodontitis (JP) in 40 adolescents in Nigeria, ${ }^{22}$ observed that all 20 patients with JP had Rhesus positive blood group $B$ or $A B$. The remaining sample of non-JP shows Rh-negative of $\mathrm{B}, \mathrm{O}$, or $\mathrm{AB}$ blood groups. Another paper of 45 individuals discovered that 15 of the patients had AP that was most usually detected in patients with $\mathrm{AB}$ blood type (60\%) or O blood type (40\%). ${ }^{21}$ Correspondingly, patients with periodontitis showed the highest ratio (57.14\%) in blood group $\mathrm{AB}$ among the total subjects of $100 .^{23}$ According to a reported study, ${ }^{16}$ more than $46.7 \%$ of the samples were affected having blood group A. A study showed a relatively high percentage of blood group B (20.97\%) in AP patients. ${ }^{24}$ Findings from the studies suggest that there is a possible genetic reason for the association of PD with $\mathrm{ABO}$ blood groups; however, from the small sample size of the study, it is not harder to conclude conclusively about the relationship of this link

\section{Relationship of ABO with the Incidence of Chronic Periodontitis}

Few studies identified the association of the ABO blood group and the occurrence of $\mathrm{CP}$, while other studies were unable to find such association. ${ }^{1,16,17}$ These differences across research were believed to be caused by the geographic diversity among people. Four of our studied papers in this review ${ }^{25-28}$ acknowledged the link between $O$ blood group and periodontitis, whereas three pieces of research ${ }^{29}$ identified a link between blood group $\mathrm{B}$ and periodontitis. ${ }^{30}$

The $61-\mathrm{kD}$ bacterial adhesion protein is a key receptor for the blood type antigen $\mathrm{H}$, which results in the increased sensitivity of blood group $\mathrm{O}$ to peptic ulcers. ${ }^{31}$ For B and A 


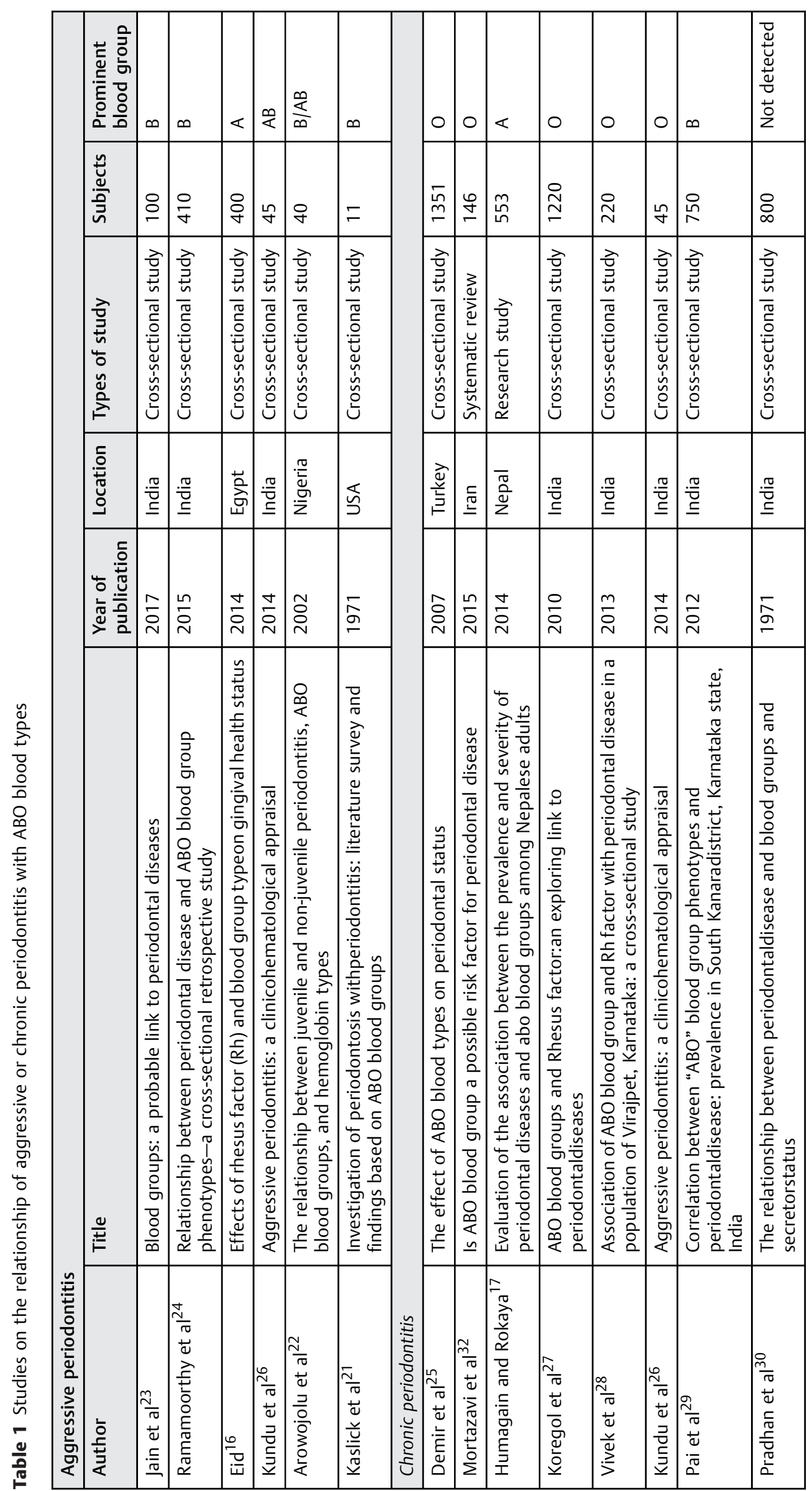


antigens to be produced, an $\mathrm{H}$ antigen is a precursor for them. The precursor $\mathrm{H}$ antigen in persons belonging to $\mathrm{A}$ and $\mathrm{B}$ antigens (antigens belonging to $\mathrm{A}$ and $\mathrm{B}$ ) is covered up to $\mathrm{A}$ and $B$ antigens, whereas in the blood group $O$ persons, it is left unexpanded. That results in the largest quantity of $\mathrm{H}$ antigen seen in persons with blood type 0 . Hence, persons with blood group $\mathrm{O}$ have a higher level of $\mathrm{H}$ antigen in their gingival tissues; these issues may be involved in the receptor process for gram-negative species. ${ }^{31,32}$ Expression of blood type antigens also depends on keratinized and unterminated epithelial differentiating patterns. Keratinized squamous epithelia occasionally express antigen A or B, while precursor $\mathrm{H}$-antigen is mostly expressed by the spinal cells. Maximum spinous expressing cells are antigen A and B compared with precursor $\mathrm{H}$ in a nonkeratinized epithelium, including the mouth mucosa. A/B transferases and their substrates in oral tissues determine the expression of $\mathrm{A} / \mathrm{B}$ antigens. ${ }^{17,33}$

\section{Future Perspective}

This review showed that six of the researches provided here showed that the blood type 0 can serve as a prognostic factor for the development of PD. However, we propose the adoption of a multicentric collaborative study in the future that would encompass a diverse range of populations and ethnicities and determine whether there is any genetic linkage on a molecular level. Investigations of the interactions between the RBC surface antigens and gingival epithelial cells with new technology may give new insight into the relationship between $\mathrm{ABO}$ blood types and PD. Anecdotal evidence suggesting a probable association between blood types and PD might be confirmed by using an animal's model for the $\mathrm{ABO}$ gene. According to the research findings from this literature review, this link has yet to be thoroughly examined.

\section{Conclusion}

From the findings of this review, it can be concluded that the ABO blood types and Rhesus factor can be a risk factor for the development of PD. The outcomes of the review reveal a possible connection between the $\mathrm{ABO}$ blood group and periodontitis. The generated data can be utilized as a steppingstone to narrow the study and target the most sensitive individuals, and then to concentrate on devising specific treatment plans for those patients.

\section{Conflict of Interest}

None.

\section{References}

1 Kinane DF, Stathopoulou PG, Papapanou PN. Periodontal diseases. Nat Rev Dis Primers 2017;3(01):1-14

2 Listgarten MA. A perspective on periodontal diagnosis. J Clin Periodontol 1986;13(03):175-181

3 Gotsman I, Lotan C, Soskolne WA, et al. Periodontal destruction is associated with coronary artery disease and periodontal infection with acute coronary syndrome. J Periodontol 2007;78(05): 849-858
4 Jeffcoat MK, Hauth JC, Geurs NC, et al. Periodontal disease and preterm birth: results of a pilot intervention study. J Periodontol 2003;74(08):1214-1218

5 Khader YS, Dauod AS, El-Qaderi SS, Alkafajei A, Batayha WQ. Periodontal status of diabetics compared with nondiabetics: a meta-analysis. J Diabetes Complications 2006;20(01):59-68

6 Lang NP, Lindhe J. Clinical Periodontology and Implant Dentistry, 2 Volume Set. West Sussex, UK: John Wiley \& Sons; 2015

7 Kassebaum NJ, Bernabé E, Dahiya M, Bhandari B, Murray CJ, Marcenes W. Global burden of severe periodontitis in 19902010: a systematic review and meta-regression. J Dent Res 2014;93(11):1045-1053

8 Holtfreter B, Albandar JM, Dietrich T, et al; Joint EU/USA Periodontal Epidemiology Working Group. Standards for reporting chronic periodontitis prevalence and severity in epidemiologic studies: proposed standards from the Joint EU/USA Periodontal Epidemiology Working Group. J Clin Periodontol 2015;42(05): 407-412

9 Offenbacher S. Periodontal diseases: pathogenesis. Ann Periodontol 1996;1(01):821-878

10 Watkins WM. The ABO blood group system: historical background. Transfus Med 2001;11(04):243-265

11 Skaik YAM. ABO blood groups and myocardial infarction among Palestinians. Ann Card Anaesth 2009;12(02):173-174

12 Biswas J, Islam MA, Rudra S, et al. Relationship between blood groups and coronary artery disease. Mymensingh Med J 2008;17 (2, Suppl):S22-S27

13 Whincup PH, Cook DG, Phillips AN, Shaper AG. ABO blood group and ischaemic heart disease in British men. BMJ 1990;300 (6741):1679-1682

14 Mitchell JRA. An association between abo blood-group distribution and geographical differences in death-rates. Lancet 1977;1 (8006):295-297

15 Stakishă̌tis DV, Ivashkiavichene LI, Narvilene AM. [Atherosclerosis of the coronary arteries and the blood group in the population of Lithuania]. Vrach Delo 1991;(08):55-57

16 Eid $\mathrm{H}$. Effects of Rhesus factor (Rh) and blood group type on gingival health status. Egypt Dent J 2011;57(04):1-6

17 Humagain M, Rokaya D. Evaluation of association between the prevalence and severity of periodontal diseases and Abo blood groups among Nepalese adults. Indian J Sci Technol 2014;23(06): 66-70

18 Baer PN. The case for periodontosis as a clinical entity. J Periodontol 1971;42(08):516-520

19 Taubman MA, Valverde P, Han X, Kawai T. Immune response: the key to bone resorption in periodontal disease. J Periodontol 2005; 76:2033-2041

20 Sofaer JA. Genetic approaches in the study of periodontal diseases. J Clin Periodontol 1990;17(7 Pt 1):401-408

21 Kaslick RS, Chasens AI, Tuckman MA, Kaufman B. Investigation of periodontosis with periodontitis: literature survey and findings based on ABO blood groups. J Periodontol 1971;42(07):420-427

22 Arowojolu MO, Dosmu EB, Adingbola TS. The relationship between juvenile and non-juvenile periodontitis, $\mathrm{ABO}$ blood groups and haemoglobin types. Afr J Med Med Sci 2002;31(03):249-252

23 Jain A, Devaraj C, Kuckreja H, Kuckreja KBS. Blood Groups: a probable link to periodontal diseases. Dental J Advance Studies 2017;5(01):47-50

24 Ramamoorthy B, Varghese SS, Ramesh A. Relationship between periodontal disease and abo blood group phenotypes-a cross sectional retrospective study. Int J Phramacy Pharmaceutical Sci 2015;7(11):386

25 Demir T, Tezel A, Orbak R, Eltas A, Kara C, Kavrut F. The effect of ABO blood types on periodontal status. Eur J Dent 2007;1(03): 139-143

26 Kundu D, Bandyopadhyay P, Nair V, Chowdhury M, Mukherjee S, Nayek M. Aggressive periodontitis: a clinico-hematological appraisal. J Indian Soc Periodontol 2014;18(02):166-171 
27 Koregol AC, Raghavendra M, Nainegali S, Kalburgi N, Varma S. ABO blood groups and Rhesus factor: an exploring link to periodontal diseases. Indian J Dent Res 2010;21(03): 364-368

28 Vivek S, Jain J, Simon SP, Battur H, Supreetha S, Haridas R. Association of $\mathrm{ABO}$ Blood Group and Rh factor with periodontal disease in a population of Virajpet, Karnataka: a cross-sectional study. J Int Oral Health 2013;5(04):30-34

29 Pai GP, Dayakar MM, Shaila M, Dayakar A. Correlation between "ABO" blood group phenotypes and periodontal disease: prevalence in south Kanara district, Karnataka state, India. J Indian Soc Periodontol 2012;16(04):519
30 Pradhan AC, Chawla TN, Samuel KC, Pradhan S. The relationship between periodontal disease and blood groups and secretor status. J Periodontal Res 1971;6(04):294-300

$31 \mathrm{Al}$ Ghamdi ASJMS. Association between ABO blood groups and severity of chronic periodontitis. Magalat Game'at al-Malik Abdul Aziz Al-U'lum al-Tibyat 2009;16(03):31-41

32 Mortazavi H, Lotfi G, Fadavi E, Hajian S, Baharvand M, Sabour S. Is ABO blood group a possible risk factor for periodontal disease? Dent Hypotheses 2015;6(01):14-18

33 Campi C, Escovich L, Valdés V, et al. Secretor status and ABH antigens expression in patients with oral lesions. Med Oral Patol Oral Cir Bucal 2007;12(06):E431-E434 